\title{
Capillary telangiectasia mimicking an active inflammatory MS-lesion in repetitive cMRIs
}

\author{
Michael Harzheim' \\ Claudia Gemmel' \\ Bernd Sommer ${ }^{2}$ \\ Ulf Kallweit' \\ Dieter Pöhlau' \\ 'MS Center, Department \\ of Neurology, Kamillus-Klinik, \\ Asbach, Germany; ${ }^{2}$ Radiologische \\ Gemeinschaftspraxis, Bad Honnef, \\ Germany
}

\begin{abstract}
Repetitive cranial magnetic resonance imaging (MRI) showed lesions typical of multiple sclerosis (MS) with one Gd-enhancing focus in the left parietal cerebrum which correlated to the clinical symptoms of a secondary progressive female MS patient. Since this Gd-enhancing lesion lasted over more than two years even after numerous intravenous high-dose methylprednisolone administrations and intrathecal triamcinolone injections the inflammatory etiology of this lesion was questioned and T2* echo-gradient images and MR angiography showed a capillary telangiectasia mimicking an active inflammatory MS-lesion.
\end{abstract}

Keywords: multiple sclerosis, capillary telangiectasia, cMRI

In multiple sclerosis (MS) patients Gd-enhancing foci in cMRI are usually interpreted as active inflammatory lesions and corticosteroid therapy is supposed to repair the destroyed blood brain barrier resulting in a loss of $\mathrm{Gd}$ enhancement in control cMRI whereas Gd enhancement will last in vascular malformations such as capillary telangiectasia, which also show increased signal intensity on T2 weighted images and usually are small, solitary, and benign in their clinical manifestation. ${ }^{1-3}$

Here we report on a secondary progressive female MS patient presenting with a right-side accentuated severe spastic tetraparesis. The diagnosis was made according to McDonald and colleagues (2001). The time since first diagnosis of initially relapsingremitting MS was three years whereas first symptoms due to MS occurred some years earlier. Initially, the patient received an immunomodulatory therapy with interferon beta- $1 \mathrm{~b}$ subcutaneously for one year. Because of high frequent relapses and the beginning of a continuous deterioration the therapy was escalated. An immunosuppressive therapy with mitoxantrone $20 \mathrm{mg}$ every three months was administered 12 times $\left(129.3 \mathrm{mg} / \mathrm{m}^{2}\right)$. Because of further clinical decline, cMRI was performed to estimate the inflammatory activity showing lesions typical of MS with one Gd-enhancing focus in the left parietal cerebrum which correlated to the right-side accentuated tetraparesis. Repetitive cMRIs after two months, 24 months, 25 months, and 27 months performed under enlarged treatment regimen (intrathecal administration of triamcinolone $(2 \times 40 \mathrm{mg}$ every three months), continuous intrathecal baclofen administration via a pump system, local intramuscular botulinum toxine injections) supplemented by intravenous high-dose methylprednisolone administration every three months showed a lasting lesion in the left parietal cerebrum without any change in shape and size and persistent $\mathrm{Gd}$ enhancement as a marker for an active inflammatory process.

As clinical findings were not suitable just to the left hemispheric lesion, continuous deterioration took place, and Gd enhancement persisted over more than two years even after high-dose corticosteroid treatment $\mathrm{T} 2 *$ echo-gradient images and MR angiography were performed and capillary telangiectasia was diagnosed (Figure 1). 


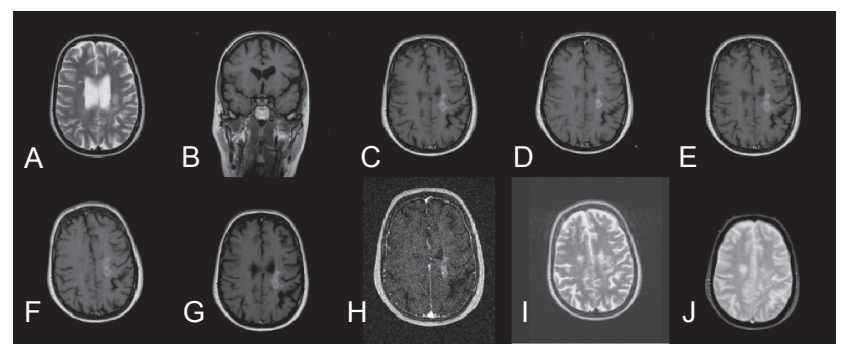

Figure I Magnetic resonance imaging (MRI) scans of our patient. A: Month 0, T2 weighted MRI; B: Month 0, TI weighted MRI; C: Month 0,TI weighted MRI + Gd; D: Month 2,TI weighted MRI + Gd; E: Month 24,TI weighted MRI + Gd; F: Month 25, TI weighted MRI + Gd; G: Month 27,TI weighted MRI + Gd; H: Month 27, MRA; I: Month 27,T2 weighted MRI; J: Month 27,T2* echo-gradient MRI.

In MS patients, Gd-enhancing lesions in cMRI should, especially when they show persistence of Gd enhancement over a long period and under therapy with corticosteroids, not be only considered as MS-associated even if clinical examination and lesion site are compatible. The coincidence of MS and vascular malformation or tumors in the central nervous system has been rarely described but otherwise vascular malformations are often mistakenly diagnosed as MS. ${ }^{1}$ In our case we found a singular vascular malformation of the brain without any findings, which indicated a complex syndrome such as macrocephaly-capillary malformation. ${ }^{5,6}$ Echo-gradient $\mathrm{T} 2 *$ images showing a very low intensity signal whereas $\mathrm{T} 2$ sequences showed an high intensity signal led to the diagnosis of capillary telangiectasia. ${ }^{6}$

\section{Disclosure}

The authors report no conflicts of interest in this work.

\section{References}

1. Tang SC, Jeng JS, Liu HM, Yip PK. Diffuse capillary telangiectasia of the brain manifested as a slowly progressive course. Cerebrovasc Dis. 2003;15:140-142.

2. Stahl SM, Johnson KP, Malamud N. The clinical and pathological spectrum of brain-stem vascular malformations. Long-term course simulates multiple sclerosis. Arch Neurol. 1980;37:25-29.

3. Yoshida Y, Terae S, Kudo K, Tha KK, Imamura M, Miyasaka K. Capillary telangiectasia of the brain stem diagnosed by susceptibilityweighted imaging. J Comput Assist Tomogr. 2006;30:980-982.

4. Conway RL, Pressman BD, Dobyns WB, et al. Neuroimaging findings in macrocephaly-apillary malformation: a longitudinal study of 17 patients. Am J Med Genet A. 2007;143:2981-3008.

5. Castillo M, Morrison T, Shaw JA, Bouldin TW. MR imaging and histologic features of capillary telangiectasia of the basal ganglia. AJNR Am J Neuroradiol. 2001;22:1553-1555.

6. Auffray-Calvier E, Desal HA, Freund P, Laplaud D, Mathon G, De Kersaint-Gilly A. Capillary telangiectasis, angiographically occult vascular malformations. MRI symptomatology apropos of 7 cases. J Neuroradiol. 1999;26:257-261. 